\title{
A NOTE ON FREIMMAN'S THEOREM IN VECTOR SPACES
}

\author{
TOM SANDERS
}

\begin{abstract}
A famous result of Freiman describes the sets $A$, of integers, for which $|A+A| \leq K|A|$. In this short note we address the analagous question for subsets of vector spaces over $\mathbb{F}_{2}$. Specifically we show that if $A$ is a subset of a vector space over $\mathbb{F}_{2}$ with $|A+A| \leq K|A|$ then $A$ is contained in a coset of size at most $2^{O\left(K^{3 / 2} \log K\right)}|A|$, which improves upon the previous best, due to Green and Ruzsa, of $2^{O\left(K^{2}\right)}|A|$. A simple example shows that the size may need to be at least $2^{\Omega(K)}|A|$.
\end{abstract}

\section{Introduction}

If $A$ and $B$ are subsets of an abelian group $G$ then we define the sumset, $A+B$, to be the set of all elements formed by adding an element of $A$ to an element of $B$ i.e. $A+B=\{a+b: a \in A$ and $b \in B\}$. There is a famous result of Frelman Fre73] which in some sense describes the sets $A \subset \mathbb{Z}$ for which $|A+A| \leq K|A|$. This note concerns what in modern parlance would be called the finite field analogue of Freimman's result. Specifically it concerns the following theorem.

Theorem 1.1. (Finite Field Freıman) Suppose that $G$ is a vector space over $\mathbb{F}_{2}$. Suppose that $A \subset G$ is a finite set with $|A+A| \leq K|A|$. Then $A$ is contained in a coset of size at most $f(K)|A|$.

While finite field models are an important tool for understanding problems in general abelian groups this result has independent significance in coding theory and has been pursued by a number of authors. We do not attempt a comprehensive survey here, but mention a few papers which are important from our standpoint.

The paper DHP04 of Deshouillers, Hennecart and Plagne provides an overview of the problem and records a quantitative version of Theorem 1.1 due to Ruzsa; it is a relatively simple argument which shows that one may take $f(K) \leq K 2^{\left\lfloor K^{3}\right\rfloor-1}$. The bulk of their paper concerns refined estimates for the case when $K$ is small; by contrast our interest lies in the asymptotics.

In a recent paper of Green and Ruzsa GR06 the authors improve Ruzsa's bound from [DHP04] when they show that one may take $f(K) \leq K^{2} 2^{\left\lfloor 2 K^{2}-2\right\rfloor}$. In this note we refine this further by proving that one may take $f(K) \leq 2^{O\left(K^{3 / 2} \log K\right)}$. Formally, then, we shall prove the following theorem.

Theorem 1.2. Suppose that $G$ is a vector space over $\mathbb{F}_{2}$. Suppose that $A \subset G$ is a finite set with $|A+A| \leq K|A|$. Then $A$ is contained in a coset of size at most $2^{O\left(K^{3 / 2} \log K\right)}|A|$.

For comparison we record the following well known example. Let $H$ be a finite subgroup of $G$ and $g_{1}+H, \ldots, g_{K-1}+H$ be $K-1$ linearly independent cosets of 
$H$ in the quotient space $G / H$. Let $A$ be the union of $H$ and the representatives $g_{1}, \ldots, g_{K-1}$. Then $|A|=|H|+K-1 \sim|H|$ and

$$
|A+A|=K(|H|+(K-1) / 2) \sim K|H| \lesssim K|A| .
$$

However $A$ contains a linearly independent set of size $\operatorname{dim} H+K-1$ and so $A$ is not contained in a coset of dimension less than $\operatorname{dim} H+K-2$ hence if $H^{\prime}$ is a coset containing $A$ then

$$
\left|H^{\prime}\right| \geq 2^{\operatorname{dim} H+K-2}=2^{K-2}|H| \gtrsim 2^{K-2}|A| .
$$

This example perhaps suggests that one could take $f(K) \leq 2^{O(K)}$ in Theorem 1.1. In fact there are other more compelling reasons to believe this, however it does not seem to reflect the underlying situation; in Gre05. Green addresses this concern by introducing (a special case of) the Polynomial FreĬman-Ruzsa conjecture (attributed to Marton in Ruz99) which, if true, seems to have some very important applications. For a detailed discussion of this see either the paper of Green or Chapter 5 of the book [TV06] of Tao and Vu.

\section{Proof of Theorem 1.2}

In [GR07, Green and Ruzsa extended Ruzsa's proof of Freľman's theorem from Ruz94 to arbitrary abelian groups; for an exposition see Gre02. Our proof is a refinement of theirs.

Their method becomes significantly simpler in the vector space setting, and would immediately give us the following weak version of the main theorem.

Theorem 2.1. Suppose that $G$ is a vector space over $\mathbb{F}_{2}$. Suppose that $A \subset G$ is a finite set with $|A+A| \leq K|A|$. Then $A$ is contained in a coset of size at most $2^{O\left(K^{2} \log K\right)}|A|$.

The proof involves three main step.

- (Finding a good model) First we use the fact that $|A+A| \leq K|A|$ to show that $A$ can be embedded as a dense subset of $\mathbb{F}_{2}^{n}$ in a way which preserves much of its additive structure.

- (Bogolubov's argument) Next we show that if $A$ is a dense subset of a compact vector space over $\mathbb{F}_{2}$ and $A+A$ is not much bigger than $A$ then $2 A-2 A$ contains a large subspace.

- (Pullback and covering) Finally we use our embedding to pull back this subspace to a coset in the original setting. A covering argument then gives us the result.

In the remainder of the note we follow through this programme with our refinement occurring at the second stage.

2.2. Finding a good model. The appropriate notion of structure preserving was introduced by Freimman in Fre73]; we record the definition now. If $G$ and $G^{\prime}$ are two abelian groups containing the sets $A$ and $A^{\prime}$ respectively then we say that $\phi: A \rightarrow A^{\prime}$ is a Freiman s-homomorphism if whenever $a_{1}, \ldots, a_{s}, b_{1}, \ldots, b_{s} \in A$ satisfy

$$
a_{1}+\ldots+a_{s}=b_{1}+\ldots+b_{s}
$$

we have

$$
\phi\left(a_{1}\right)+\ldots+\phi\left(a_{s}\right)=\phi\left(b_{1}\right)+\ldots+\phi\left(b_{s}\right) .
$$


If $\phi$ has an inverse which is also an $s$-homomorphism then we say that $\phi$ is a Freiman s-isomorphism.

A simple but elegant argument of Green and Ruzsa establishes the existence of a small vector space into which we can embed our set via a Freĭman isomorphism. Specifically they prove the following proposition.

Proposition 2.3. (Proposition 6.1, GR07) Suppose that $A$ is a subset of a vector space over $\mathbb{F}_{2}$. Suppose that $|A+A| \leq K|A|$. Then there is a vector space $G^{\prime}$ over $\mathbb{F}_{2}$ with $\left|G^{\prime}\right| \leq K^{2 s}|A|$, a set $A^{\prime} \subset G^{\prime}$, and a Frěman s-isomorphism $\phi: A \rightarrow A^{\prime}$.

2.4. Bogolyubov's argument. In this section we show that if $A$ is a subset of a compact vector space over $\mathbb{F}_{2}$ then $2 A-2 A$ contains a large subspace. Originally (in Ruz94) Ruzsa employed an argument of Bogolubov with the Fourier transform. This was refined by Chang in Cha02, and the improvement of this note rests on a further refinement. We shall need some notation for the Fourier transform and we record this now; Rudin, Rud90, includes all the results which we require.

Suppose that $G$ is a compact vector space over $\mathbb{F}_{2}$. Write $\widehat{G}$ for the dual group, that is the discrete vector space over $\mathbb{F}_{2}$ of continuous homomorphisms $\gamma: G \rightarrow S^{1}$, where $S^{1}:=\{z \in \mathbb{C}:|z|=1\}$. $G$ may be endowed with Haar measure $\mu_{G}$ normalised so that $\mu_{G}(G)=1$ and as a consequence we may define the Fourier transform $\widehat{\imath}: L^{1}(G) \rightarrow \ell^{\infty}(\widehat{G})$ which takes $f \in L^{1}(G)$ to

$$
\widehat{f}: \widehat{G} \rightarrow \mathbb{C} ; \gamma \mapsto \int_{x \in G} f(x) \gamma(-x) d \mu_{G}(x) .
$$

In Cha02] Chang proved the following result. (Although in Cha02] it is stated for $\mathbb{Z} / N \mathbb{Z}$, the same proof applies to any compact abelian group and in particular to compact vector spaces over $\mathbb{F}_{2}$.)

Proposition 2.5. Suppose that $G$ is a compact vector space over $\mathbb{F}_{2}$. Suppose that $A \subset G$ has density $\alpha$ and $\mu_{G}(A+A) \leq K \mu_{G}(A)$. Then $2 A-2 A$ contains (up to a null set) a subspace of codimension $O\left(K \log \alpha^{-1}\right)$.

We prove the following refinement of this.

Proposition 2.6. Suppose that $G$ is a compact vector space over $\mathbb{F}_{2}$. Suppose that $A \subset G$ has density $\alpha$ and $\mu_{G}(A+A) \leq K \mu_{G}(A)$. Then $2 A-2 A$ contains (up to a null set) a subspace of codimension $O\left(K^{1 / 2} \log \alpha^{-1}\right)$.

To prove this we require the following pure density version of the proposition.

Proposition 2.7. (Theorem 2.4, San08) Suppose that $G$ is a compact vector space over $\mathbb{F}_{2}$. Suppose that $A \subset G$ has density $\alpha$. Then $2 A-2 A$ contains (up to a null set) a subspace of codimension $O\left(\alpha^{-1 / 2}\right)$.

The proof in $\underline{\operatorname{San} 08}$ is significantly simpler in the vector space setting. Since the ideas are important we include the proof here; the basic technique is iterative.

Lemma 2.8. (Iteration lemma) Suppose that $G$ is a compact vector space over $\mathbb{F}_{2}$. Suppose that $A \subset G$ has density $\alpha$. Then at least one of the following is true.

(i) $2 A-2 A$ contains all of $G$ (up to a null set).

(ii) There is a subspace $V$ of $\widehat{G}$ with dimension 1, an element $x \in G$ and a set $A^{\prime} \subset V^{\perp}$ with the following properties.

- $x+A^{\prime} \subset A$; 


$$
\text { - } \mu_{V^{\perp}}\left(A^{\prime}\right) \geq \alpha\left(1+2^{-1} \alpha^{1 / 2}\right) \text {. }
$$

Proof. As usual with problems of this type studying the sumset $2 A-2 A$ is difficult so we turn instead to $g:=\chi_{A} * \chi_{A} * \chi_{-A} * \chi_{-A}$ which has support equal to $2 A-2 A$. One can easily compute the Fourier transform of $g$ in terms of that of $\chi_{A}$ :

$$
\widehat{g}(\gamma)=|\widehat{\chi A}(\gamma)|^{4} \text { for all } \gamma \in \widehat{G},
$$

from which it follows that $g$ is very smooth. Specifically $\widehat{g} \in \ell^{\frac{1}{2}}(\widehat{G})$ since

$$
\begin{aligned}
\sum_{\gamma \in \widehat{G}}|\widehat{g}(\gamma)|^{\frac{1}{2}} & =\sum_{\gamma \in \widehat{G}}|\widehat{\chi A}(\gamma)|^{2} \\
& =\alpha \text { by Parseval's theorem. }
\end{aligned}
$$

We may assume that $\mu_{G}(2 A-2 A)<1$ since otherwise we are in the first case of the lemma, so $S:=(2 A-2 A)^{c}$ has positive density, say $\sigma$. Plancherel's theorem gives

$$
0=\left\langle\chi_{S}, g\right\rangle=\sum_{\gamma \in \widehat{G}} \overline{\widehat{\chi_{S}}(\gamma)} \widehat{g}(\gamma) \Rightarrow\left|\widehat{\chi_{S}}\left(0_{\widehat{G}}\right) \widehat{g}\left(0_{\widehat{G}}\right)\right| \leq \sum_{\gamma \neq 0_{\widehat{G}}}\left|\widehat{\chi_{S}}(\gamma) \widehat{g}(\gamma)\right| .
$$

$\widehat{g}\left(0_{\widehat{G}}\right)=\alpha^{4}, \widehat{\chi_{S}}\left(0_{\widehat{G}}\right)=\sigma$ and $\left|\widehat{\chi_{S}}(\gamma)\right| \leq\left\|\chi_{S}\right\|_{1}=\sigma$, so the above yields

$$
\sigma \alpha^{4} \leq \sigma \sum_{\gamma \neq 0_{\widehat{G}}}|\widehat{g}(\gamma)| \Rightarrow \alpha^{4} \leq \sum_{\gamma \neq 0_{\widehat{G}}}|\widehat{g}(\gamma)| \text { since } \sigma>0 .
$$

Finding a non-trivial character at which $\widehat{g}$ is large is now simple since $\widehat{g} \in \ell^{\frac{1}{2}}(\widehat{G})$.

$$
\alpha^{4} \leq \sup _{\gamma \neq 0_{\widehat{G}}}|\widehat{g}(\gamma)|^{\frac{1}{2}}\left(\sum_{\gamma \in \widehat{G}}|\widehat{g}(\gamma)|^{\frac{1}{2}}\right) \leq \sup _{\gamma \neq 0_{\widehat{G}}}|\widehat{\chi A}(\gamma)|^{2} \cdot \alpha
$$

by (2.1). Rearranging this we have

$$
\sup _{\gamma \neq 0_{\widehat{G}}}|\widehat{\chi A}(\gamma)| \geq \alpha^{\frac{3}{2}} .
$$

The set $\Gamma:=\left\{\gamma \in \widehat{G}:|\widehat{\chi A}(\gamma)| \geq \alpha^{\frac{3}{2}}\right\}$ has size at most $\alpha^{-2}$ since

$$
|\Gamma| \cdot\left(\alpha^{\frac{3}{2}}\right)^{2} \leq \sum_{\gamma \in \widehat{G}}|\widehat{\chi A}(\gamma)|^{2}=\alpha \text { by Parseval's theorem. }
$$

It follows that the supremum in (2.2) is really a maximum and we may pick a character $\gamma$ which attains this maximum. We now proceed with a standard $L^{\infty}$ density-increment argument. Let $V:=\left\{0_{\widehat{G}}, \gamma\right\}$ and $f:=\chi_{A}-\alpha$. Then

$$
\int f * \mu_{V^{\perp}} d \mu_{G}=0 \text { and }\left\|f * \mu_{V^{\perp}}\right\|_{1} \geq\left\|\widehat{f} \widehat{\mu_{V^{\perp}}}\right\|_{\infty}=\left|\widehat{\chi_{A}}(\gamma)\right| .
$$

Adding these we conclude that

$$
\begin{aligned}
\left|\widehat{\chi \chi_{A}}(\gamma)\right| & \leq 2 \int\left(f * \mu_{V^{\perp}}\right)_{+} d \mu_{G} \\
& =2 \int\left(\chi_{A} * \mu_{V^{\perp}}-\alpha\right)_{+} d \mu_{G} \\
& \leq 2\left(\left\|\chi_{A} * \mu_{V^{\perp}}\right\|_{\infty}-\alpha\right) .
\end{aligned}
$$


$\chi_{A} * \mu_{V^{\perp}}$ is continuous so there is some $x \in G$ with

$$
\chi_{A} * \mu_{V^{\perp}}(x)=\left\|\chi_{A} * \mu_{V^{\perp}}\right\|_{\infty} \geq \alpha\left(1+2^{-1} \alpha^{1 / 2}\right) .
$$

The result follows on taking $A^{\prime}=x+A$.

Proof of Proposition 2.7. We define a nested sequence of finite dimensional subspaces $V_{0} \leq V_{1} \leq \ldots \leq \widehat{G}$, elements $x_{k} \in V_{k}^{\perp}$ and subsets $A_{k}$ of $V_{k}^{\perp}$ with density $\alpha_{k}$, such that $x_{k}+A_{k} \subset A_{k-1}$. We begin the iteration with $V_{0}:=\left\{0_{\widehat{G}}\right\}, A_{0}:=A$ and $x_{0}=0_{G}$.

Suppose that we are at stage $k$ of the iteration. If $\mu_{V_{k}^{\perp}}\left(2 A_{k}-2 A_{k}\right)<1$ then we apply Lemma 2.8 to $A_{k}$ considered as a subset of $V_{k}^{\perp}$. We get a vector space $V_{k+1}$ with $\operatorname{dim} V_{k+1}=1+\operatorname{dim} V_{k}$, an element $x_{k+1} \in G$ and a set $A_{k+1}$ such that

$$
x_{k+1}+A_{k+1} \subset A_{k} \text { and } \alpha_{k+1} \geq \alpha_{k}\left(1+2^{-1} \alpha_{k}^{1 / 2}\right) .
$$

It follows from the density increment that if $m_{k}=2 \alpha_{k}^{-1 / 2}$ then $\alpha_{k+m_{k}} \geq 2 \alpha_{k}$. Define the sequence $\left(N_{l}\right)_{l}$ recursively by $N_{0}=0$ and $N_{l+1}=m_{N_{l}}+N_{l}$. The density $\alpha_{N_{l}}$ is easily estimated:

$$
\alpha_{N_{l}} \geq 2^{l} \alpha \text { and } N_{l} \leq \sum_{s=0}^{l} 2 \alpha_{N_{s}}^{-1 / 2} \leq 2 \alpha^{-1 / 2} \sum_{s=0}^{l} 2^{-s / 2}=O\left(\alpha^{-1 / 2}\right)
$$

Since density cannot be greater than 1 there is some stage $k$ with $k=O\left(\alpha^{-1 / 2}\right)$ when the iteration cannot proceed i.e. for which $2 A_{k}-2 A_{k}$ contains all of $V_{k}^{\perp}$ (except for a null set). By construction of the $A_{k} \mathrm{~s}$ there is a translate of $A_{k}$ which is contained in $A_{0}=A$ and hence $2 A_{k}-2 A_{k}$ is contained in $2 A-2 A$. It follows that $2 A-2 A$ contains (up to a null set) a subspace of $G$ of codimension $k=O\left(\alpha^{-1 / 2}\right)$.

The key ingredient in the proof of Proposition 2.6 is the following iteration lemma, which has a number of similarities with Lemma 2.8 .

Lemma 2.9. Suppose that $G$ is a compact vector space over $\mathbb{F}_{2}$. Suppose that $A, B \subset G$ have $\mu_{G}(A+B) \leq K \mu_{G}(B)$. Write $\alpha$ for the density of $A$. Then at least one of the following is true.

(i) B contains (up to a null set) a subspace of codimension $O\left(K^{1 / 2}\right)$.

(ii) There is a subspace $V$ of $\widehat{G}$ with dimension 1, elements $x, y \in G$ and sets $A^{\prime}, B^{\prime} \subset V^{\perp}$ with the following properties.

- $x+A^{\prime} \subset A$ and $y+B^{\prime} \subset B$;

- $\mu_{V \perp}\left(A^{\prime}\right) \geq \alpha\left(1+2^{-3 / 2} K^{-1 / 2}\right)$;

- $\mu_{V \perp}\left(A^{\prime}+B^{\prime}\right) \leq K \mu_{V \perp}\left(B^{\prime}\right)$.

Proof. If $\mu_{G}(B) \geq(2 K)^{-1}$ then we apply Proposition 2.7 to get that $B$ contains (up to a null set) a subspace of codimension $O\left(K^{1 / 2}\right)$ and we are in the first case of the lemma. Hence we assume that $\mu_{G}(B) \leq(2 K)^{-1}$. 
Write $\beta$ for the density of $B$. We have

$$
\begin{aligned}
(\alpha \beta)^{2} & =\left(\int \chi_{A} * \chi_{B} d \mu_{G}\right)^{2} \\
& \leq \mu_{G}(A+B) \int\left(\chi_{A} * \chi_{B}\right)^{2} d \mu_{G} \text { by Cauchy-Schwarz, } \\
& \leq K \beta \int\left(\chi_{A} * \chi_{B}\right)^{2} d \mu_{G} \text { by hypothesis, } \\
& =K \beta \sum_{\gamma \in \widehat{G}}\left|\widehat{\chi_{A}}(\gamma)\right|^{2}\left|\widehat{\chi_{B}}(\gamma)\right|^{2} \text { by Parseval's theorem. }
\end{aligned}
$$

The main term in the sum on the right is the contribution from the trivial character, in particular

$$
\left|\widehat{\chi A}\left(0_{\widehat{G}}\right)\right|^{2}\left|\widehat{\chi_{B}}\left(0_{\widehat{G}}\right)\right|^{2}=\alpha^{2} \beta^{2}
$$

while

$$
\begin{aligned}
\sum_{\gamma \neq 0_{\widehat{G}}}\left|\widehat{\chi_{A}}(\gamma)\right|^{2}\left|\widehat{\chi_{B}}(\gamma)\right|^{2} & \leq \sup _{\gamma \neq 0_{\widehat{G}}}\left|\widehat{\chi_{A}}(\gamma)\right|^{2} \sum_{\gamma \in \widehat{G}}\left|\widehat{\chi_{B}}(\gamma)\right|^{2} \\
& =\beta \sup _{\gamma \neq 0_{\widehat{G}}}\left|\widehat{\chi_{A}}(\gamma)\right|^{2} \text { by Parseval's theorem for } \chi_{B} .
\end{aligned}
$$

Putting these last two observations in (2.3) gives

$$
\alpha^{2} \beta^{2} \leq K \beta^{3} \alpha^{2}+K \beta^{2} \sup _{\gamma \neq 0_{\widehat{G}}}|\widehat{\chi A}(\gamma)|^{2} .
$$

Since $K \beta \leq 2^{-1}$ we can rearrange this to conclude that

$$
\sup _{\gamma \neq 0_{\widehat{G}}}|\widehat{\chi A}(\gamma)| \geq(2 K)^{-1 / 2} \alpha \text {. }
$$

The set $\Gamma:=\left\{\gamma \in \widehat{G}:\left|\widehat{\chi_{A}}(\gamma)\right| \geq(2 K)^{-1 / 2} \alpha\right\}$ has size at most $2 K \alpha^{-1}$ since

$$
|\Gamma| \cdot\left((2 K)^{-1 / 2} \alpha\right)^{2} \leq \sum_{\gamma \in \widehat{G}}\left|\widehat{\chi_{A}}(\gamma)\right|^{2}=\alpha \text { by Parseval's theorem. }
$$

It follows that the supremum in (2.4) is really a maximum and we may pick a character $\gamma$ which attains this maximum. We now proceed with a standard $L^{\infty}$ density-increment argument. Let $V:=\left\{0_{\widehat{G}}, \gamma\right\}$ and $f:=\chi_{A}-\alpha$. Then

$$
\int f * \mu_{V^{\perp}} d \mu_{G}=0 \text { and }\left\|f * \mu_{V^{\perp}}\right\|_{1} \geq\left\|\widehat{f} \widehat{\mu_{V^{\perp}}}\right\|_{\infty}=\left|\widehat{\chi_{A}}(\gamma)\right| .
$$

Adding these we conclude that

$$
\begin{aligned}
|\widehat{\chi A}(\gamma)| & \leq 2 \int\left(f * \mu_{V^{\perp}}\right)_{+} d \mu_{G} \\
& =2 \int\left(\chi_{A} * \mu_{V^{\perp}}-\alpha\right)_{+} d \mu_{G} \\
& \leq 2\left(\left\|\chi_{A} * \mu_{V^{\perp}}\right\|_{\infty}-\alpha\right) .
\end{aligned}
$$

Since $\chi_{A} * \mu_{V^{\perp}}$ is continuous it follows that there is some $x$ for which

$$
\chi_{A} * \mu_{V^{\perp}}(x) \geq \alpha\left(1+2^{-3 / 2} K^{-1 / 2}\right) .
$$


Let $x^{\prime}+V^{\perp}:=G \backslash\left(x+V^{\perp}\right)$ be the other coset of $V^{\perp}$ in $G$. Write $A_{1}=A \cap(x+$ $\left.V^{\perp}\right), B_{1}=B \cap\left(x+V^{\perp}\right)$ and $B_{2}=B \cap\left(x^{\prime}+V^{\perp}\right)$. Now $A_{1} \subset A$ so

$$
\left(A_{1}+B_{1}\right) \cup\left(A_{1}+B_{2}\right) \subset A+B_{1} \cup B_{2},
$$

and $A_{1}+B_{1} \subset V^{\perp}$ while $A_{1}+B_{2} \subset x+x^{\prime}+V^{\perp}$ so these two sets are disjoint and we conclude that

$$
\begin{aligned}
\mu_{G}\left(A_{1}+B_{1}\right)+\mu_{G}\left(A_{1}+B_{2}\right) & =\mu_{G}\left(\left(A_{1}+B_{1}\right) \cup\left(A_{1}+B_{2}\right)\right) \\
& \leq \mu_{G}\left(A+B_{1} \cup B_{2}\right) \\
& \leq K \mu_{G}\left(B_{1} \cup B_{2}\right) \text { by hypothesis } \\
& \leq K\left(\mu_{G}\left(B_{1}\right)+\mu_{G}\left(B_{2}\right)\right) .
\end{aligned}
$$

Hence, by averaging, there is some $i$ such that

$$
\mu_{G}\left(A_{1}+B_{i}\right) \leq K \mu_{G}\left(B_{i}\right) .
$$

We take $A^{\prime}=x+A_{1}$ and, if $i=1, B^{\prime}=x+B_{1}$ and $y=x$, while if $i=2$, $B^{\prime}=x^{\prime}+B_{2}$ and $y=x^{\prime}$. The result follows.

Proof of Proposition 2.6. We define a nested sequence of finite dimensional subspaces $V_{0} \leq V_{1} \leq \ldots \leq \widehat{G}$, elements $x_{k}, y_{k} \in V_{k}^{\perp}$, and subsets $A_{k}$ and $B_{k}$ of $V_{k}^{\perp}$ such that $A_{k}+x_{k} \subset A_{k-1}$ and $B_{k}+y_{k} \subset B_{k-1}$ and $\mu_{V_{k}^{\perp}}\left(A_{k}+B_{k}\right) \leq K \mu_{V_{k}^{\perp}}\left(B_{k}\right)$. We write $\alpha_{k}$ for the density of $A_{k}$ in $V_{k}^{\perp}$. Begin the iteration with $V_{0}:=\left\{0_{\widehat{G}}\right\}$, $B_{0}=A_{0}:=A$ and $x_{0}=y_{0}=0_{G}$.

Suppose that we are at stage $k$ of the iteration. We apply Lemma 2.9 to $A_{k}$ and $B_{k}$ inside $V_{k}^{\perp}$ (which we can do since $\mu_{V_{k}^{\perp}}\left(A_{k}+B_{k}\right) \leq K \mu_{V_{k}^{\perp}}\left(B_{k}\right)$ ). It follows that either $2 B_{k}-2 B_{k}$ contains (up to a null set) a subspace of codimension $O\left(K^{1 / 2}\right)$ in $V_{k}^{\perp}$ or we get a subspace $V_{k+1} \leq \widehat{V_{k}}$ with $\operatorname{dim} V_{k+1}=1+\operatorname{dim} V_{k}$, elements $x_{k+1}, y_{k+1} \in V_{k}^{\perp}$ and sets $A_{k+1}$ and $B_{k+1}$ with the following properties.

- $x_{k+1}+A_{k+1} \subset A_{k}$ and $y_{k+1}+B_{k+1} \subset B_{k}$;

- $\mu_{V^{\perp}}\left(A_{k+1}\right) \geq \alpha_{k}\left(1+2^{-3 / 2} K^{-1 / 2}\right)$;

- $\mu_{V^{\perp}}\left(A_{k+1}+B_{k+1}\right) \leq K \mu_{V^{\perp}}\left(B_{k+1}\right)$.

It follows from the density increment that if $m=2^{3 / 2} K^{1 / 2}$ then $\alpha_{k+m} \geq 2 \alpha_{k}$, and hence the iteration must terminate (because density can be at most 1 ) at some stage $k$ with $k=O\left(K^{1 / 2} \log \alpha^{-1}\right)$. The iteration terminates if $2 B_{k}-2 B_{k}$ contains (up to a null set) a subspace of codimension $O\left(K^{1 / 2}\right)$ in $V_{k}^{\perp}$, from which it follows that $2 A-2 A \supset 2 B_{k}-2 B_{k}$ contains (up to a null set) a subspace of codimension $k+O\left(K^{1 / 2}\right)=O\left(K^{1 / 2} \log \alpha^{-1}\right)$.

2.10. Pullback and covering. We now complete the proof of the main theorem using a covering argument.

We are given $A \subset G$ finite with $|A+A| \leq K|A|$. By Proposition 2.3 there is a finite vector space $G^{\prime}$ with $\left|G^{\prime}\right| \leq K^{16}|A|$ and a subset $A^{\prime}$ with $A^{\prime}$ Freimman 8-isomorphic to $A$. It follows that

$$
\mu_{G^{\prime}}\left(A^{\prime}\right) \geq K^{-16} \text { and } \mu_{G^{\prime}}\left(A^{\prime}+A^{\prime}\right) \leq K \mu_{G^{\prime}}\left(A^{\prime}\right) .
$$

We apply Proposition 2.6 to conclude that $2 A^{\prime}-2 A^{\prime}$ contains a subspace of codimension $O\left(K^{1 / 2} \log K\right)$. However, $A$ is 8 -isomorphic to $A^{\prime}$ so $2 A-2 A$ is 2 -isomorphic 
to $2 A^{\prime}-2 A^{\prime}$ and it is easy to check that the 2-isomorphic pullback of a subspace is a coset so $2 A-2 A$ contains a coset of size

$$
2^{-O\left(K^{1 / 2} \log K\right)}\left|G^{\prime}\right| \geq 2^{-O\left(K^{1 / 2} \log K\right)}|A| .
$$

The following covering result of Chang [Cha02] converts this large coset contained in $2 A-2 A$ into a small coset containing $A$. It is true in more generality than we state; we only require the version below.

Proposition 2.11. Suppose that $G$ is a vector space over $\mathbb{F}_{2}$. Suppose that $A \subset G$ is a finite set with $|A+A| \leq K|A|$. Suppose that $2 A-2 A$ contains a coset of size $\eta|A|$. Then $A$ is contained in a coset of size at most $2^{O\left(K \log K \eta^{-1}\right)}|A|$.

Theorem 1.2 follows immediately from this proposition and the argument preceding it.

\section{CONCLUDing REMARKS}

It is worth making a couple of concluding remarks. First, all the implicit constants in the work are effective however they are not particularly neat or significant so it does not seem to be important to calculate them. Secondly, and more importantly, it seems likely that one could modify Proposition 2.6 to fall within the more general framework of approximate groups pioneered by Bourgain in Bou99. It does not seem that this would lead to any improvement in Frelman's theorem for $\mathbb{Z}$, essentially because of the need to narrow the Bohr sets at each stage of the iteration.

\section{ACKNOWLEDGEMENTS}

I should like to thank Tim Gowers and Ben Green for encouragement and supervision.

\section{REFERENCES}

[Bou99] J. Bourgain. On triples in arithmetic progression. Geom. Funct. Anal., 9(5):968-984, 1999.

[Cha02] M.-C. Chang. A polynomial bound in Frel̆man's theorem. Duke Math. J., 113(3):399419, 2002.

[DHP04] J.-M. Deshouillers, F. Hennecart, and A. Plagne. On small sumsets in $(\mathbb{Z} / 2 \mathbb{Z})^{n}$. Combinatorica, 24(1):53-68, 2004.

[Fre73] G. A. Freĭman. Foundations of a structural theory of set addition. American Mathematical Society, Providence, R. I., 1973. Translated from the Russian, Translations of Mathematical Monographs, Vol 37.

[GR06] B. J. Green and I. Z. Ruzsa. Sets with small sumset and rectification. Bull. London Math. Soc., 38(1):43-52, 2006.

[GR07] B. J. Green and I. Z. Ruzsa. Frelman's theorem in an arbitrary abelian group. J. Lond. Math. Soc. (2), 75(1):163-175, 2007.

[Gre02] B. J. Green. Edinburgh-MIT lecture notes on Freĭman's theorem. http://www.dpmms.cam.ac.uk/ bjg23, 2002.

[Gre05] B. J. Green. Notes on the polynomial Freĭman-Ruzsa conjecture. http://www.dpmms.cam.ac.uk/ bjg23, 2005.

[Rud90] W. Rudin. Fourier analysis on groups. Wiley Classics Library. John Wiley \& Sons Inc., New York, 1990. Reprint of the 1962 original, A Wiley-Interscience Publication.

[Ruz94] I. Z. Ruzsa. Generalized arithmetical progressions and sumsets. Acta Math. Hungar., 65(4):379-388, 1994.

[Ruz99] I. Z. Ruzsa. An analog of Freĭman's theorem in groups. Astérisque, (258):xv, 323-326, 1999. Structure theory of set addition. 
[San08] T. Sanders. Additive structures in sumsets. Math. Proc. Cambridge Philos. Soc., 144(2):289-316, 2008.

[TV06] T. C. Tao and H. V. Vu. Additive combinatorics, volume 105 of Cambridge Studies in Advanced Mathematics. Cambridge University Press, Cambridge, 2006.

Department of Pure Mathematics and Mathematical Statistics, University of CamBridge, Wilberforce Road, Cambridge CB3 0WA, England

E-mail address: t.sanders@dpmms.cam.ac.uk 